INPLASY

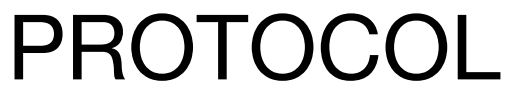

To cite: An et al. Effectiveness comparisons of Chinese patent medicine on sciatica A protocol for systematic review and Bayesian network metaanalysis. Inplasy protocol 2020110073. doi:

10.37766/inplasy2020.11.0073

Received: 17 November 2020

Published: 17 November 2020

Corresponding author:

Pengfei Zhu

zpf1061643414@163.com

Author Affiliation:

Xintai Affiliated Hospital of

Shandong First Medical

University

Support: 2012GGB14067 ; SDYJG19144.

Review Stage at time of this submission: Preliminary searches.

Conflicts of interest: We declare no conflicts of interest.

\section{Effectiveness comparisons of Chinese patent medicine on sciatica A protocol for systematic review and Bayesian network meta-analysis}

Review question / Objective: To evaluate the efficacy and safety of Chinese patent medicine (CPM) for sciatica by means of systematic review and network meta-analysis (NMA), and to compare them in order.

Condition being studied: Sciatica is one of the common clinical diseases. Studies have proved the efficacy of Chinese patent medicine (CPM) in the treatment of sciatica, so far, there has not been a complete systematic review of its effectiveness and safety, and the comparative efficacy and safety of CPM have not been ranked. Therefore, it is necessary to evaluate the efficacy and safety of these CPM by means of systematic review and network meta-analysis (NMA), and to compare them in order.

INPLASY registration number: This protocol was registered with the International Platform of Registered Systematic Review and Meta-Analysis Protocols (INPLASY) on 17 November 2020 and was last updated on 17 November 2020 (registration number INPLASY2020110073).

\section{INTRODUCTION}

Review question / Objective: To evaluate the efficacy and safety of Chinese patent medicine (CPM) for sciatica by means of systematic review and network metaanalysis (NMA), and to compare them in order.
Condition being studied: Sciatica is one of the common clinical diseases. Studies have proved the efficacy of Chinese patent medicine (CPM) in the treatment of sciatica, so far, there has not been a complete systematic review of its effectiveness and safety, and the comparative efficacy and safety of CPM 
have not been ranked. Therefore, it is necessary to evaluate the efficacy and safety of these CPM by means of systematic review and network metaanalysis (NMA), and to compare them in order.

\section{METHODS}

Search strategy: Two researchers will independently search PubMed, Cochrane Library, EMbase, Web of Science, CNKI, Wanfang, VIP, CBM and other databases for RCTs of CPM in the treatment of sciatica, (database established until December 30, 2020). In addition, manually search the "Pharmaceutical Information", "National Essential Drug List", "Chinese Pharmacopoeia", etc. to inquire about drug instructions, and screen the market circulation and clinically commonly used CPM. Formulate search strategies based on the characteristics of different databases, and use a combination of Medical Subject Headings (MeSH) and free words.

Participant or population: We will include patients with clearly diagnosed sciatica according to the diagnostic criteria of sciatica issued by National Institute for Health and Clinical Excellence (NICE) . Patients in different intervention groups in the same study will have no significant imbalances in baseline conditions such as age and gender, which is comparable.

Intervention: The patients in the experimental group will be treated with a kind of CPM or combined with western medicine.

Comparator: The patients in the control group will be treated with routine western medicine, including non-steroidal antiinflammatory drugs, muscle relaxants, glucocorticoids, nutritional nerve drugs, etc.

Study designs to be included: We will include the random control trails (RCTs) of CPM published at home and abroad for the treatment of sciatica, and the language will be limited to Chinese and English.
Eligibility criteria: Patients diagnosed with sciatica.

Information sources: We will search PubMed, Cochrane Library, EMbase, Web of Science, CNKI, Wanfang, VIP, CBM, "Pharmaceutical Information", "National Essential Drug List", "Chinese Pharmacopoeia" to collect RCT for sciatica.

Main outcome(s): The primary outcome indicator will be the clinical efficacy, which can be divided into four levels: (1) Recovery: the pain disappeared completely, straight leg elevation test (-), return to normal work; (2) Significant effect: the pain basically disappeared, the straight leg elevation test (-), but after strenuous exercise, the affected area still feels uncomfortable; (3) Improvement: the pain is significantly reduced, the straight leg elevation test is more than 60 degrees, and the affected area feels sore whenever overworked; (4) Invalid: there is no significant change in the condition after 1 course of treatment.

Additional outcome(s): The secondary outcome indicators will be pain degree and lumbar spine function: (1) Pain degree: Visual Analogue Score (VAS) [11] is a pain evaluation method commonly used in clinical, which is sensitive and easy for patients to judge. Make a table with equal scales from "0" to "10". A score of "0" indicates that the patient has no pain, and a score of "10" indicates that the patient feels severe pain and is unbearable. The degree of pain gradually increases with the increase of the scale, allowing the patient to select the corresponding scale according to their true feeling of pain before and after treatment, and the tester truthfully records the pain score. (2) Lumbar spine function: Japanese Orthopaedic Association (JOA) Score [12] was created by the Japanese Orthopaedic Association and is a standard for evaluating lumbar spine function. It mainly evaluate patients' clinical symptoms, signs and activities of daily living. Patients are scored before and after treatment. The higher the score, the more obvious the 
recovery of activity function, the lower the score, the worse the recovery of activity. (3) adverse events.

Quality assessment / Risk of bias analysis: Two researchers will evaluate the risk of bias in the included studies in accordance with Cochrane Handbook 5.1.0 [13], including: (1 )The method of random sequence generation; (2) Whether the allocation plan is hidden; (3) Whether the subject and researcher are blinded; (4) Whether the outcome assessor is blinded; (5) Whether the result data is complete; (6) Whether to report the research results selectively; (7) Other sources of bias. In case of disagreement, the corresponding author shall make a ruling. According to the results of the evaluation, the Review Manager 5.3 software will be used to make a risk of bias chart for the included studies.

Strategy of data synthesis: RevMan software will be used for bias evaluation and heterogeneity test. Binary variables will use odds ratio (OR) as the effect indicator, and continuous variables will use mean difference (MD) or standardized mean difference (SMD) as the effect indicator, with $95 \%$ confidence interval and $P<0.05$ used as the standard of statistical difference. The degree of heterogeneity will be judged by 12 . If $P \geq 0.1$ and $I 2 \leq 50 \%$, it means that the heterogeneity between the studies is small, and the fixed effects model will be used, followed by a NMA; if $P<0.1$ and $12>50 \%$, we will analyze the source of heterogeneity, use subgroup analysis or sensitivity analysis to deal with obvious clinical heterogeneity, eliminate heterogeneity factors or use random effects model to merge analysis, and use descriptive analysis if the source of heterogeneity cannot be found. Use the R software gemtc package to draw the network diagram. The GeMTC software will be used for NMA and draw the surface under cumulative ranking area (SUCRA). We will use Markov chain-Monte Carlo (MCMC) for Bayesian inference and random effects model for analysis. We will set the initial value, chain number, iteration, annealing, and step length corresponding parameters and calculate. The inconsistency test will adopt the node splitting method. As for $\mathbf{P}>\mathbf{0 . 0 5}$ for each study in the subgroup, we will adopt the consistency model, otherwise adopt the inconsistency model. Model convergence will be reflected by potentialscalereduced factor (PSRF). When PSRF is equal to or close to 1 , the convergence performance is better and the analysis results of the model are more reliable.

Subgroup analysis: If $P<0.1$ and $12>50 \%$, we will analyze the source of heterogeneity, use subgroup analysis or sensitivity analysis to deal with obvious clinical heterogeneity, eliminate heterogeneity factors or use random effects model to merge analysis, and use descriptive analysis if the source of heterogeneity cannot be found.

Sensibility analysis: If $\mathbf{P}<\mathbf{0 . 1}$ and $\mathbf{I} 2>50 \%$, we will analyze the source of heterogeneity, use subgroup analysis or sensitivity analysis to deal with obvious clinical heterogeneity, eliminate heterogeneity factors or use random effects model to merge analysis, and use descriptive analysis if the source of heterogeneity cannot be found.

\section{Country(ies) involved: China.}

Keywords: sciatica; Chinese patent medicine; network meta-analysis; protocol.

Contributions of each author:

Author 1 - Hongqiang An.

Author 2 - Bing Li.

Author 3 - Jifeng Zhao.

Author 4 - Zhijian Ao.

Author 5 - Xiaohui Zhong.

Author 6 - Pengfei Zhu.

Author 7 - Jianlin Wu. 